\title{
FREE PRODUCTS OF HOPFIAN LATTICES
}

Dedicated to the memory of Hanna Neumann

(Received 3rd January 1973)

G. GRÄTZER and J. SICHLER

Communicated by M. F. Newman

\section{Introduction}

In this paper we are going to prove the following results:

THEOREM 1. There exist two bounded hopfian lattices such that their $\{0,1\}-$ free product is not hopfian.

THEOREM 2. There exist two hopfian lattices such that their free product is not hopfian.

In Theorem 2 free product (coproduct, sum) has its usual meaning (see, for instance, [4]); in Theorem 1 we use the usual definition but all lattices are assumed to be bounded (that is, having a least element 0 and largest element 1) and all homomorphisms are assumed to be $\{0,1\}$-homomorphisms (that is, homomorphisms preserving 0 and 1 ).

Recall, that a lattice $L$ (group, ring, and so on) is called hopfian if $L$ is not isomorphic to any proper quotient $L / \Theta$ (proper means that $\Theta \neq \omega$ ); or equivalently, if any onto endomorphism of $L$ is an automorphism. A remarkable result of Evans [3] states that every finitely presentable lattice is hopfian. A finitely generated nonhopfian lattice has recently been found by Wille (unpublished).

This paper grew out of a colloquium lecture of H. Neumann in which she reported on hopfian groups, in particular on the result of [2], namely that the free product of two finitely generated hopfian groups is hopfian. The problem whether this is true in general was left open. Theorem 2 of this paper gives a negative answer to this question for lattices. Later it was shown by the second author [13] that a modification of the set theoretic scheme of this paper (see section 3 ) could be used to settle the original group theoretic problem.

Both authors were supported by the National Research Council of Canada. 
The lattices we construct for Theorem 2 are all $>\aleph_{0}$ generated. It is an open problem whether this result could be improved. Could the two lattices be constructed to be $\aleph_{0}$-generated; one finitely generated, one $\aleph_{0}$-generated; one finitely presented, one $\aleph_{0}$-generated; both finitely generated; or the ultimate: one finitely generated and the other finitely presented? The last two possibilities are ruled out if the following conjecture is true: the free product of two bounded hopfian lattices is hopfian again.

The basic idea of the construction is to use a set theoretic scheme which provides two "hopfian objects" whose union is not hopfian. The technical work in section 3 converts this scheme into two hopfian graphs whose disjoint union is not hopfian.

Graphs can be turned into lattices using our earlier paper [7]; this conversion preserves the properties of being hopfian and being non-hopfian, while the disjoint union of graphs is transformed into the $\{0,1\}$-free product of the corresponding lattices. Thus the results of section 3 yield Theorem 1 . To obtain Theorem 2 it is necessary to do some more work about free products of lattices and this is accomplished in section 4. All the lattice theoretic lemmas are proved in section 2 .

\section{Lattice theoretic lemmas}

By a graph $\langle X ; R\rangle$ we mean a nonvoid set $X$ with a symmetric binary relation $R$ such that $\{a, a\} \in R$ for no $a \in X$ (that is, an undirected graph with no loops). A triangle of $\langle X ; R\rangle$ is a three element subset $\left\{a_{0}, a_{1}, a_{2}\right\}$ of $X$ such that $\left\{a_{0}, a_{1}\right\},\left\{a_{1}, a_{2}\right\},\left\{a_{2}, a_{0}\right\} \in R$. All graphs in this paper will be assumed to have the property that every vertex is contained in at least one triangle.

Let $\langle X ; R\rangle$ be a graph, let $F_{0,1}(X)$ be the bounded lattice freely generated by $X$, and let $\Theta$ be the smallest congruence relation on $F_{0,1}(X)$ such that $x \wedge y$ $\equiv 0(\Theta)$ and $x \vee y \equiv 1(\Theta)$ for all $\{x, y\} \in R$. The lattice $M(X, R)=F_{0,1}(X) / \Theta$ constructed in [7] has the following properties:

(i) $X \subseteq M(X, R)$ and no two distinct elements of $X$ are comparable in $M(X, R)$;

(ii) $X$ generates $M(X, R)$;

(iii) $\{a, b\}$ is a complemented pair in $M(X, R)$ if and only if either $\{a, b\}$ $=\{0,1\}$ or $\{a, b\} \in R$ (this follows from [1] or [5]).

Since $M(X, R)$ is the "most free" lattice satisfying (i)-(iii), every compatible mapping $\phi:\langle X ; R\rangle \rightarrow\langle Y ; S\rangle$ extends uniquely to a $\{0,1\}$-homomorphism $M(\phi): M(X, R) \rightarrow M(Y, S)$; in [7] we proved that every $\{0,1\}$-homomorphism $f: M(X, R) \rightarrow M(Y, S)$ is equal to $M(\phi)$ for some compatible mapping $\phi:\langle X ; R\rangle$ $\rightarrow\langle Y ; S\rangle$.

The lattice $M(X, R)$ can also be described as a subset of $F_{0.1}(X)$ for which (iv) if $a, b \in M(X, R)$ then $a \leqq b$ in $M(X, R)$ if and only if $a \leqq b$ in $F_{0,1}(X)$;

(v) the 0 and 1 of $M(X, R)$ is the 0 and 1 of $F_{0.1}(X)$, respectively; 

or $[5])$.

(vi) if $a, b \in M(X, R)-\{0,1\}$, then $a \equiv b(\Theta)$ if and only if $a=b$ (see [1]

(vii) if $a, b, c \in M(X, R)-\{0,1\}$ and if $c$ is a lower bound of $\{a, b\}$, then $a \wedge b$ formed in $F_{0.1}(X)$ lies in $M(X, R)$ and is equal to the meet of $a, b$ in $M(X, R)$; dually for the join.

Now we start proving our lemmas.

LEMMA 1. $X$ is the set of all join and meet-irreducible elements of $M(X, R)$.

Proof. It is known (Whitman [14]) that any $x \in X$ is irreducible in $F_{0.1}(X)$. If $x=y \vee z$ in $M(X, R), y \neq x, z \neq x$, then $\{y, z\}$ is disjoint with $\{0,1\}$ and so $x=y \vee z$ in $F_{0}(X)$ by (vii), a contradiction. It follows from (ii) that no other element is irreducible.

Let $M_{5}$ denote the five element modular nondistributive lattice, see Figure 1. If $\left\{a_{0}, a_{1}, a_{2}\right\}$ is a triangle in $\langle X ; R\rangle$, then $\left\{0, a_{0}, a_{1}, a_{2}, 1\right\}$ is a sublattice isomorphic to $M_{5}$; we call it the sublattice associated with the triangle $\left\{a_{0}, a_{1}, a_{2}\right\}$.

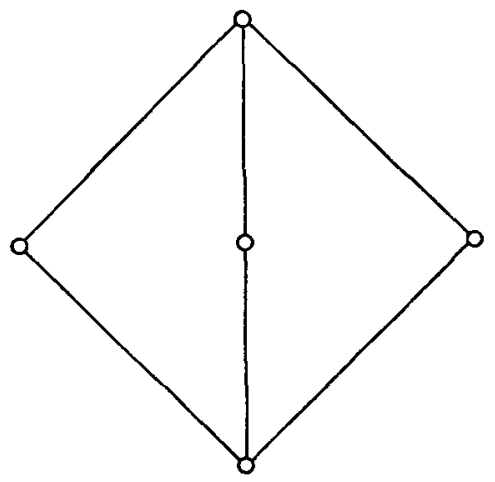

Figure 1

The following result is implicit in [12]:

LeMmA 2. A sublattice of $M(X, R)$ is isomorphic with $M_{5}$ if and only if it is associated with a triangle of $\langle X ; R\rangle$.

Proof. "If" being trivial we prove "only if". Let $\{a, a, b, c, i\}$ be a sublattice of $M(X, R)$ isomorphic to $M_{5}$ with the bounds $o$ and $i$. If $o=0$ and $i=1$, then by (iii), $\{a, b, c\}$ is a triangle of $\langle X ; R\rangle$ as required. If $o \neq 0$ and $i \neq 1$, then by (vii), $\{o, a, b, c, i\}$ is also a sublattice of $F_{0,1}(X)$ which is known ([10] and [14]) not to have such sublattices. Finally, let $o \neq 0$ and $i=1$ (or dually). Then $o=a \wedge b=a \wedge c$ in $F_{0,1}(X)$ by (vii). Therefore, by a result of Jónsson [10], $o=a \wedge(b \vee c)$ in $F_{0.1}(X)$. Since $b \vee c \equiv 1(\Theta)$ we conclude that $a \equiv o(\Theta)$, contradicting (vi). 
COROllary 3. $M(X, R)$ has no sublattice isomorphic to the lattice of Figure 2 or to $M_{5} \times 2$.

Proof. Indeed, if $\left\{0, a_{1}, a_{2}, b, c, i\right\}$ is a sublattice isomorphic to the lattice of Figure 2, then by Lemma $2,\left\{a_{i}, b, c\right\}$ is a triangle for $i=1,2$ and so $a_{1}, a_{2} \in X$. Since $a_{1}<a_{2}$ this contradicts (i). Also, if a sublattice is isomorphic to $M_{5} \times 2$, then we find a sublattice isomorphic to $M_{5}$ not including $\{0,1\}$, contradicting Lemma 2.

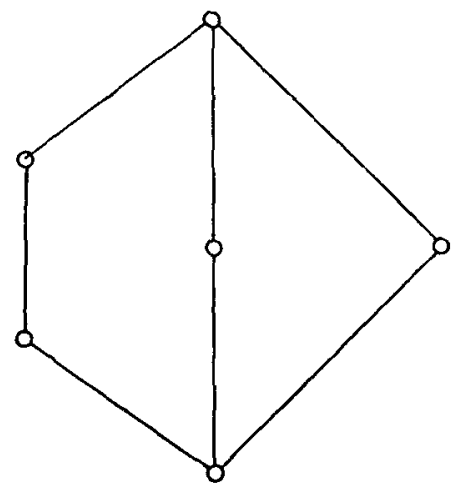

Figure 2

Call a graph $\langle X ; R\rangle$ hopfian if every compatible map of $\langle X ; R\rangle$ onto itself is an automorphism.

LEMMA 4. $\langle X ; R\rangle$ is hopfian if and only if $M(X, R)$ is hopfian.

Proor. Let $\langle X ; R\rangle$ be hopfian and let $f$ be an onto endomorphism of $M(X, R)$. Then there exists a compatible map $\phi: X \rightarrow X$ such that $f=M(\phi)$. If $\phi$ is not onto, then $x \notin \phi(X)$ for some $x \in X$. But $f(M(X, R))$ is generated by $\phi(X)$ by (ii), $x$ is irreducible by Lemma 1 , hence $x \notin f(M(X, R))$, contradicting that $f$ is onto. Therefore $\phi$ is onto. Since $\langle X ; R\rangle$ is hopfian, $\phi$ is an automorphism and so there is another automorphism $\phi^{\prime}$ such that $\phi \phi^{\prime}$ and $\phi^{\prime} \phi$ are the identity map on $X$. Thus, both $M\left(\phi \phi^{\prime}\right)=M(\phi) M\left(\phi^{\prime}\right)$ and $M\left(\phi^{\prime} \phi\right)=M\left(\phi^{\prime}\right) M(\phi)$ are equal to the identity map of $M(X, R)$ and so $f=M(\phi)$ is an automorphism, which was to be proved. Conversely, if $\langle X ; R\rangle$ is not hopfian, then there is a compatible onto map $\phi: X \rightarrow X$ that is not an automorphism. Now if $M(X, R)$ is hopfian, then $M(\phi)$ is an automorphism, hence it has an inverse $M\left(\phi^{\prime}\right)$. Just as before, $\phi^{\prime}$ is an inverse of $\phi$, a contradiction. This completes the proof of the lemma.

Lemma 4 is the Reduction Theorem. Combined with the next result it completely reduces Theorem 1 to a statement on graphs.

Lemma 5. Let $\left\langle X_{i} ; R_{i}\right\rangle$ be graphs $i=1,2$, and let $\langle X ; R\rangle$ be their disjoint union. Then $M(X, R)$ is the $\{0,1\}$-free product of $M\left(X_{1}, R_{1}\right)$ and $M\left(X_{2}, R_{2}\right)$. 
Proof. Let $\phi_{i}: X_{i} \rightarrow X$ be the natural embedding of $X_{i}$ into $X$ for $i=1,2$. Then $M\left(\phi_{i}\right)$ is a $\{0,1\}$-homomorphism of $M\left(X_{i}, R_{i}\right)$ into $M(X, R)$. Now let $L$ be a bounded lattice and let $f_{i}$ be a $\{0,1\}$-homomorphism of $M\left(X_{i}, R_{i}\right)$ into $L$ for $i=1$, 2. Let $\psi_{i}$ be the restriction of $f_{i}$ to $X_{i}(i=1,2)$ and define $\psi: X \rightarrow L$ by $\psi(x)$ $=\psi_{i}(x)$ for $x \in X_{i}(i=1$ or 2$)$. If $\{x, y\} \in R$, then $\psi(x)$ and $\psi(y)$ are complementary in $L$ (since $\{x, y\} \in R$ for $n o x \in X_{1}$ and $y \in X_{2}$ ) and so there is a unique homomorphism $g: M(X, R) \rightarrow L$ extending $\psi$. Since $g M\left(\phi_{l}\right)$ and $f_{t}$ agree on $X_{i}$ we obtain $g M\left(\phi_{i}\right)=f_{i}$ for $i=1,2$. We have verified that $M(X, R)$ is the $\{0,1\}$-free product of $M\left(X_{1}, R_{1}\right)$ and $M\left(X_{2}, R_{2}\right)$.

Finally, we shall need in section 4 some results on free products. We start with a result of Lakser [11]:

LEMMA 6. Let $L_{i}, i \in I$ be lattices and let $L$ be a free product of the $L_{i}, i \in I$. Let $A$ be a sublattice of $L$ isomorphic to $M_{5}$. Then either $A \subseteq L_{i}$ for some $i \in I$ or some $L_{i}$ has a sublattice isomorphic to the lattice of Figure 2 or to $M_{5} \times 2$.

Combining Lemma 6 with Corollary 3 and Lemma 2 we obtain

COROLlaRY 7. Let $L$ be a free product of the $M\left(X_{i}, R_{i}\right), i \in I$. If $M$ is a sublattice of $L$ and $M$ is isomorphic to $M_{5}$, then $M \subseteq M\left(X_{2}, R_{i}\right)$ for some $i \in I$ and $M$ is associated with a triangle of $\left\langle X_{i} ; R_{i}\right\rangle$.

We close this section with a generalization of Lemma 1:

Lemma 8. Let $L_{i}, i \in I$ be lattices and let $L$ be a free product of the $L_{i}, i \in I$. If $a \in L_{i}$ is join-irreducible in $L_{i}$, then it is also join-irreducible in $L$ and dually.

PROof. For the proof of this lemma we have to assume that the reader is familiar with the notation and results of [6], in particular with pp. 233-235. Let $a$ be join-reducible in $L, a=b \vee c$. Let $p$ and $q$ be polynomials with $\langle p\rangle=b$, $\langle q\rangle=c$. Then $a \subseteq p \vee q$ and $p \subseteq a, q \subseteq a$. Of the rules 3.(1)-3.(6) only two may be applied to $a \subseteq p \vee q$, namely 3.(5) and 3.(2). If 3.(5) applies, then $a \subseteq p$ or $a \subseteq q$, and so $b=\langle p\rangle=a$ or $c=\langle q\rangle=a$. If $a \subseteq p \vee q$ because of 3.(2), then $a^{(j)} \leqq(p \vee q)_{(j)}=p_{(j)} \vee q_{(j)}$ for some $j \in I$. However, $a^{(1)}$ exists only for $j=i$, hence $a^{(i)}=a \leqq p_{(i)} \vee q_{(i)}$. Furthermore, $p_{(i)} \leqq a$ and $q_{(i)} \leqq a$, so $p_{(i)}$ $\vee q_{(i)}=a$. In view of the join-irreducibility of $a$ in $L_{l}$ we get $p_{(i}=a$ or $q_{(i)}=a$, say $p_{(i)}=a$. So $p_{(i)} \subseteq p \subseteq a$ and therefore $a=p_{(i)} \subseteq p \subseteq p^{(i)} \subseteq a^{(i)}=a$, yielding $b=\langle p\rangle=a$, as required.

COROLlary 9. Let L be a free product of the $M\left(X_{i}, R_{i}\right), i \in I$. Then $\bigcup\left(X_{i} \mid i \in I\right)$ can be characterized as the set of all irreducible elements of $L$.

\section{The scheme and its graph representations}

Let $N$ be the set of all positive integers and let $Z$ be the set of integers. Set

$$
C_{n}=\{n\} \times Z \text {. }
$$


If there is no danger of confusion, we write $a \in C_{n}$ for $\langle n, a\rangle$. Let us consider the sets $C_{n}$ as arranged in Figure 3. There are two arrows pointing at $C_{n}$ for each $n \in N$. The arrow $C_{2 n} \rightarrow C_{n}$ represents the map $x \mapsto 2 x$, the arrow $C_{2 n+1^{-}} \rightarrow C_{n}$ represents the map $x \mapsto 2 x+1$. For $m, n \in N$ let us define $f_{m n}: C_{m} \rightarrow C_{n}$ as follows:

(i) if $m=n$, then $f_{m m}$ is the identity map on $C_{m}$; (ii) if there is a sequence of arrows from $C_{m}$ to $C_{n}$ let $f_{m n}$ be the composition of the maps represented by the arrows; (iii) otherwise, $f_{m n}$ is not defined.
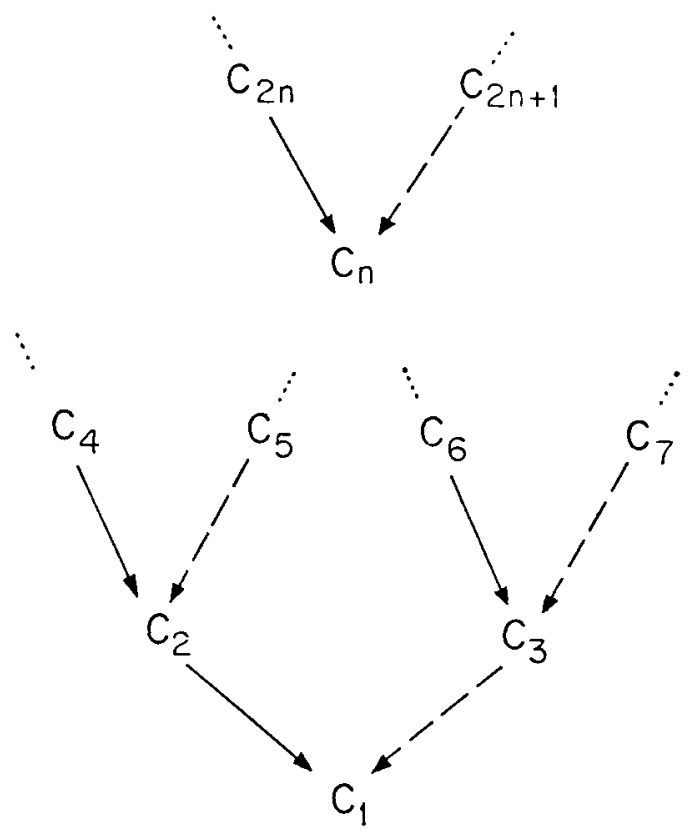

Figure 3

Observe, that between $C_{m}$ and $C_{n}$ there is at most one sequence of arrows, so if $f_{m n}$ is defined it is defined uniquely. Now set

$$
\begin{aligned}
& A_{0}=\bigcup\left(C_{2 k} \mid k \geqq 1\right), A_{1}=\bigcup\left(C_{2 k+1} \mid k \geqq 0\right), \\
& A_{2}=A_{0} \cup A_{1}=N \times Z .
\end{aligned}
$$

Call a map $g: A_{j} \rightarrow A_{j}\left(j=0,1\right.$, or 2) admissible if for any $C_{m} \subseteq A_{j}, g$ restricted to $C_{m}$ agrees with some $f_{m n}$. Call $A_{j}$ hopfian if every admissible onto map has an inverse which is also admissible, or equivalently, if the only admissible onto map is the identity map.

Lemma 10. $A_{0}$ and $A_{1}$ are hopfian but $A_{2}$ is not hopfian. 
Proof. Define $g$ on $A_{2}$ as follows: $g$ on $C_{1}$ is the identity map; $g$ is $f_{2 n . n}$ on $C_{2 n}$ for $n \geqq 1$ and $g$ is $f_{2 n+1 . n}$ on $C_{2 n+1}$ for $n \geqq 1 . g$ is onto since

$$
C_{n}=f_{2 n . n}\left(C_{2 n}\right) \cup f_{2 n+1, n}\left(C_{2 n+1}\right),
$$

for every $n \in N$. But $g$ is not one-to-one as every element of $C_{1}$ is the image of two distinct elements. Hence $A_{2}$ is not hopfian. It is somewhat more complicated to show that $A_{0}$ and $A_{1}$ are hopfian. We will show it for $A_{0}$. We start with an observation:

If $f_{n k}(x)=-1 \in C_{k}$ and $n=2 k$ or $n=2 k+1$, then $x=-1$ and $n=2 k+1$. Consequently,

$$
\text { if } f_{i k}(x)=-1 \in C_{k} \text {, then } i \text { is odd. }
$$

Now let $g: A_{0} \rightarrow A_{0}$ be an admissible onto map. We claim that $g$ is the identity map on $A_{0}$ (so we prove more than hopfian). Indeed, if $g$ is not the identity map on $A_{0}$, then there is a $k$ such that $g$ restricted to $C_{k}$ is $f_{k m}$ for some $m \neq k$. We claim that $-1 \in C_{k}$ is not in $g\left(A_{0}\right)$. Indeed, if $-1=g(x)$, then $-1=f_{n k}(x)$ for some $n \in N$ and $C_{n} \subseteq A_{0}$. But we have shown above that this would imply that $n$ is odd, contradicting $C_{n} \subseteq A_{0}$.

Observe, that -1 is the only element of $C_{k}$ not necessarily in $g\left(A_{0}\right)$; the proof of the fact that $A_{1}$ is hopfian requires 0 instead of -1 and is analogous to the proof above.

Now we start constructing the graphs. First, some definitions. Let $\langle X ; R\rangle$ be a graph. The vertices $x, y$ are said to be triangle-connected if there is a sequence $T_{0}, T_{1}, \cdots, T_{n}$ of triangles such that $x \in T_{0}, y \in T_{n}$ and $T_{i} \cap T_{i+1} \neq \phi$ for $i=0,1$, $\cdots, n-1$. Triangle-connected components of a graph and triangle-connected graphs are defined in the obvious fashion. Finally, a graph is rigid if the identity map is the only compatible map of the graph into itself. The following result is a weak version of a theorem of Hell [9]:

LEMMA 11. For every infinite cardinal $\mathrm{m}$ there exists a triangle-connected rigid graph of cardinality $\mathrm{m}$.

The idea of the transformation of the scheme into graphs is to replace each element in $C_{n}$ by a copy of an infinite triangle-connected rigid graph $\langle V ; E\rangle$ and to add some more edges so that the only compatible maps will be of the form

$$
\text { (identity on } V) \times f_{m n} \text {. }
$$

So let $\langle V ; E\rangle$ be an infinite triangle-connected rigid graph and assume that $N \subseteq V$. For every $n \in N$ we define the graph

$$
H_{n}=\left\langle V \times Z ; S_{n}\right\rangle
$$

as follows (see Figure 4 which indicates the edges listed under (b) as segments of the horizontal lines between two consecutive dots): 


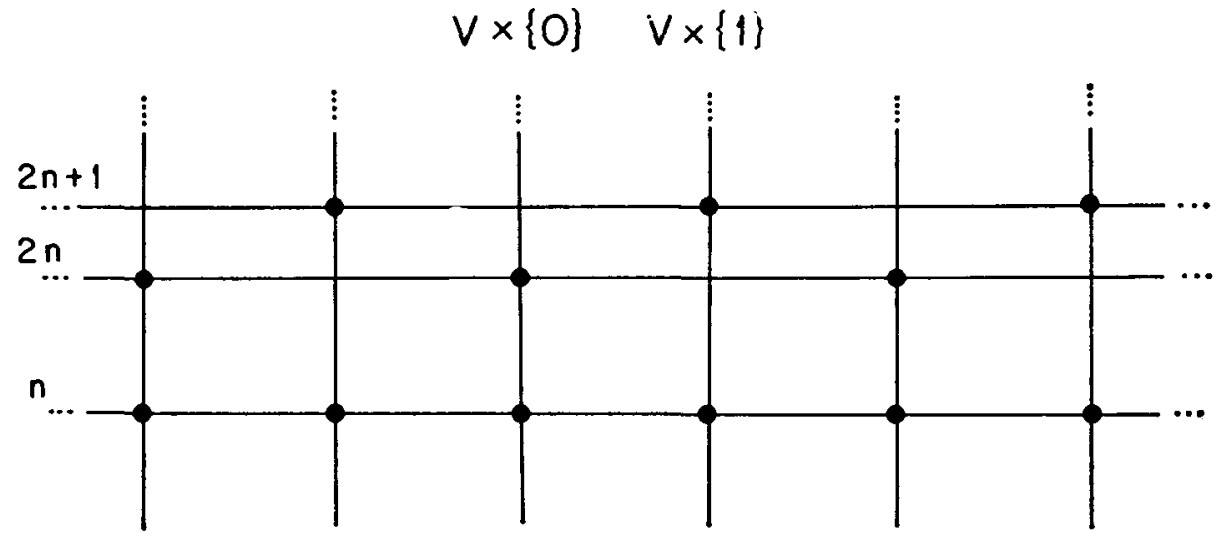

Figure 4

(a) for $i \in Z$ and $\left\{v, v^{\prime}\right\} \in E$ we set

$$
\left\{\langle v, i\rangle,\left\langle v^{\prime}, i\right\rangle\right\} \in S_{n} ;
$$

(b) for all $m \in N$ such that $f_{m n}$ is defined and for all $i \in Z$

$$
\left\{\left\langle m, f_{m n}(i)\right\rangle,\left\langle m, f_{m n}(i+1)\right\} \in S_{n} ;\right.
$$

(c) no other pairs are in $S_{n}$.

(a) shows that $V \times\{i\}$ with $S_{n}$ restricted to $V \times\{i\}$ is isomorphic to $\langle V ; E\rangle$. The edges under (b) will be called mixed. For each $m \in N$, the set $\{m\} \times Z$ is called the level $m$ of $H_{n}$. Observe that a mixed edge of $H_{n}$ is always on a level, in fact on a level $m$ with $m \geqq n$.

$H_{n}$ is connected since $\langle V ; E\rangle$ is; in fact, any two copies of $\langle V ; E\rangle$ are connected on the level $n$.

Now define the map $g_{m n}$ for all $\langle m, n\rangle$ for which $f_{m n}$ is defined:

that is,

$$
g_{m n}=(\text { identity map on } V) \times f_{m n},
$$

$$
g_{m n}\langle v, z\rangle=\left\langle v, f_{m n}(z)\right\rangle .
$$

$g_{m n}$ is obviously a compatible map of $H_{m}$ into $H_{n}$. The converse of this statement is the crucial step in this section.

LEMMA 12. Let $h$ be a compatible map of $H_{m}$ into $H_{n}$. Then $h=g_{m n}$.

ProOF. Since all mixed edges are on a level it follows easily that all the triangles of $H_{m}$ are contained in the $V \times\{i\}, i \in Z$. Thus the $V \times\{i\}, i \in Z$ are the triangle-connected components of $H_{m}$ and so they have to be mapped by $h$ into the 
triangle-connected components of $H_{n}$. Therefore, for each $i \in Z$ there exists a $\phi(i) \in Z$ such that

$$
h(V \times\{i\}) \subseteq V \times\{\phi(i)\} .
$$

Since $\langle V ; E\rangle$ is rigid we conclude that

$$
h\langle v, i\rangle=\langle v, \phi(i)\rangle,
$$

so that $\phi: Z \rightarrow Z$ determines $h$. To show $h=g_{m n}$ it will be sufficient to verify that $\phi=f_{m n}$.

Claim 1. Let $i, j \in Z, m \in N$. Then there exist $i^{\prime}, j^{\prime} \in Z$ and $m^{\prime} \in N$ such that $i^{\prime}-j^{\prime}$ is odd, and

$$
f_{m^{\prime} m}\left(i^{\prime}\right)=i \text { and } f_{m^{\prime} m}\left(j^{\prime}\right)=j .
$$

Proof. If $i-j$ is odd we set $i^{\prime}=i, j^{\prime}=j$, and $m^{\prime}=m$. Now let $i-j$ $=2^{k} \cdot t$, when $t$ is odd. We proceed by induction on $k$. If for smaller exponents the statement has been verified, then if $i$ is even, set $m^{*}=2 m$ and if $i$ is odd, set $m^{*}=2 m+1$. In both cases there are $i^{*}$ and $j^{*} \in Z$ such that $i^{*}-j^{*}=2^{k-1} \cdot t$, $f_{m^{*} m}\left(i^{*}\right)=i$, and $f_{m^{*} m}\left(j^{*}\right)=j$. Applying the induction hypothesis to $i^{*}, j^{*}, m^{*}$ we get the $i^{\prime}, j^{\prime}$, and $m^{\prime}$ as required.

ClaIM 2. $\phi$ is one-to-one.

Proof. Indeed, if $\phi(i)=\phi(j)$ for $i, j \in Z$ and $i \neq j$, then we choose $i^{\prime}, j^{\prime}$, and $m^{\prime}$ as in Claim 1. Consider the map $g=h g_{m^{\prime} m}$ of $H_{m^{\prime}}$ into $H_{n}$. Since $g_{m^{\prime} m}$ and $h$ are compatible maps so is $g$. Consider the path

$$
\left\langle m^{\prime}, i^{\prime}\right\rangle,\left\langle m^{\prime}, i^{\prime}+1\right\rangle, \cdots,\left\langle m^{\prime}, j^{\prime}\right\rangle
$$

in $H_{m}$. Any two adjacent vertices are connected by an edge since this is the level $m^{\prime}$ in $H_{m}$. Therefore,

$$
g\left\langle m^{\prime}, i^{\prime}\right\rangle, g\left\langle m^{\prime}, i^{\prime}+1\right\rangle, \cdots, g\left\langle m^{\prime}, j^{\prime}\right\rangle
$$

has the same property. Moreover,

$$
\begin{aligned}
g\left\langle m^{\prime}, i^{\prime}\right\rangle & =h\left(g_{m^{\prime} m}\left\langle m^{\prime}, i^{\prime}\right\rangle\right)=h\left\langle m^{\prime}, f_{m^{\prime} n}\left(i^{\prime}\right)\right\rangle \\
& =\left\langle m^{\prime}, \phi(i)\right\rangle=\left\langle m^{\prime}, \phi(j)\right\rangle=h\left\langle m^{\prime}, f_{m^{\prime} m}\left(j^{\prime}\right)\right\rangle=g\left\langle m^{\prime}, j^{\prime}\right\rangle .
\end{aligned}
$$

We found a cycle of an odd length on the level $m^{\prime}$ of $H_{n}$, which is impossible since any level is two-colorable.

Claim 3. $\phi(0)=f_{m n}(0)$.

Proof. $V \times\{0\}$ is distinguished in $H_{m}$ by the fact that on each level $2^{k} m$ there is a mixed edge with one vertex in $V \times\{0\}$. Therefore, $V \times\{\phi(0)\}$ has the same property in $H_{n}$ and so 


$$
\phi(0) \in f_{, k} .(C, k) \text { for } k=1,2, \cdots .
$$

So $\phi(0)=f_{m n}(x)$ where $x$ is divisible by $2^{k}$ for all $k \geqq 1$. Thus $x=0$ as claimed.

Claim 4. $\phi(1)=f_{m n}(1)$.

Proof. Same proof as that of Claim 3; use the levels $2^{k}(2 m+1)$.

Now we prove that $\phi(x)=f_{m n}(x)$ for all $x \in Z$. We already know that $\phi$ is one-to-one and $\phi(x)=f_{m n}(x)$ for $x=0$ and $x=1$. There is a mixed edge between $\langle m, 1\rangle$ and $\langle m, 2\rangle$ in $H_{m}$; therefore there is a mixed edge between $\langle m, 1\rangle$ and $\langle m, \phi(2)\rangle$ in $H_{n}$. Consequently, $\phi(2)=0$ or $\phi(2)=2$. But $\phi(2)=0$ contradicts that $\phi$ is one-to-one, hence $\phi(2)=2$. Proceeding thus, we conclude that $\phi(x)$ $=f_{m n}(x)$ for all $x \in Z$, concluding the proof of Lemma 12 .

Define $G_{0}$ as the disjoint union of the $H_{2 n}, n=1,2, \cdots$ and $G_{1}$ as the disjoint union of the $H_{2 n+1}, n=0,1,2, \cdots$. Let $G_{2}$ be the disjoint union of $G_{0}$ and $G_{1}$. Since $H_{k}$ are components of $G_{i}$, Lemma 10 and Lemma 12 combined yield that $G_{0}$ and $G_{1}$ are hopfian but $G_{2}$ is not. So if we use these graphs to construct the lattices $M\left(G_{i}\right) i=0,1,2$, by Lemmas 4 and 5 we have finished the proof of Theorem 1 .

Combining the results of [8] and [9] yields the existence of $2^{\mathrm{m}}$ pairwise nonisomorphic rigid triangle-connected graphs of any cardinality $m \geqq \kappa_{0}$. Since the correspondence $\langle X ; R\rangle \rightarrow M(X, R)$ is, in fact, a full embedding of the category of graphs into the category of bounded lattices, it follows that for every infinite $\mathfrak{m}$ there are $2^{\mathfrak{m}}$ pairs of hopfian lattices of cardinality $m$ whose $\{0,1\}$-free product is not hopfian and any two such pairs are non isomorphic. This is obviously best possible.

\section{Free products}

In this section we prove Theorem 2. Throughout the proof let $\langle V ; E\rangle$ be a fixed triangle-connected rigid graph with $|V|>\boldsymbol{N}_{0}$.

Let $K_{n}=\left\langle U_{n} ; T_{n}\right\rangle$ be defined by

$$
\begin{gathered}
U_{n}=(V \times Z) \times\{n\}, \\
\left\{\langle\langle v, z\rangle, n\rangle,\left\langle\left\langle v^{\prime}, z^{\prime}\right\rangle, n\right\rangle\right\} \in T_{n} \text { iff }\left\{\langle v, z\rangle,\left\langle v^{\prime}, z^{\prime}\right\rangle\right\} \in S_{n} .
\end{gathered}
$$

Let $h_{m n}$ be the compatible map between $K_{m}$ and $K_{n}$ corresponding to $g_{m n}$, that is, $h_{m n}\langle\langle v, z\rangle, m\rangle=\left\langle g_{m n}\langle v, z\rangle, n\right\rangle$. For $i \in N$ set

$$
B_{i}=M\left(U_{i}, T_{i}\right) \text {. }
$$

Let $L_{0}$ be the free product of the $B_{2 i}, i=1,2, \cdots$ and let $L_{1}$ be the free product of the $B_{2 i+1}, i=0,1, \cdots$. Finally, let $L$ be the free product of $L_{0}$ and $L_{1}$, or equiv. alently, of all the $B_{1}, i=1,2, \cdots$.

LEMMA 13. $L$ is not hopfian. 
Proof. Observe that $L$ is generated by $U\left(U_{i} \mid i=1,2, \cdots\right)$. Now we define a map on $L$ patterned after the map $g$ of Lemma 10 . Let $g_{1}$ be the identity on $B_{1}$ viewed as an embedding of $B_{1}$ into $L$; let $g_{2 i}$ be the extension of $g_{2 i, i}$ into a homomorphism of $B_{2 i}$ into $B_{i} \subseteq L$; let $g_{2 i+1}$ be the extension of $g_{2 i+1 i}$ into a homomorphism of $B_{2 i+1}$ into $B_{i} \subseteq L$. Since $L$ is a free product there is a homomorphism $g$ of $L$ into $L$ extending all the $g_{i}, i=1,2, \cdots$. The endomorphism $g$ is onto but not one-to-one and so $L$ is not hopfian.

LEMMA 14. $L_{0}$ is hopfian.

Proof. Let $\psi$ be an onto endomorphism of $L_{0}$. We are going to show that $\psi$ is the identity map which yields in particular that $L_{0}$ is hopfian.

$L_{0}$ is generated by $U=\bigcup\left(U_{2 i} \mid i=1,2, \cdots\right) . U_{2 i}$ is a set of irreducibles in $B_{2 i}$ and so by Lemma $8, U_{2 i}$ is a set of irreducibles of $L_{0}$. Therefore

$$
\psi(U) \supseteq U .
$$

Every $u \in U_{2 i}$ is contained in a triangle of $U_{2 i}$. If this triangle is collapsed by $\psi$ the $B_{2 i}$ is mapped into a singleton by $\psi$; otherwise, the image of the triangle yields a sublattice isomorphic to $M_{5}$ and so, by Corollary $7, \psi(u) \in U_{2 l}$ is mapped by $\psi$ into $U_{2 j}$, then $\psi\left(U_{2 i}\right) \subseteq U_{2 j}$ since the $\left\langle U_{2 k} ; T_{2 k}\right\rangle$ are all connected. If $\psi$ is not the identity map on $L_{0}$, then $\psi$ is not the identity map on some $U_{2 i}$. Since $B_{2 i}$ has no nontrivial non-constant endomorphisms, $\psi\left(U_{2 i}\right)$ is either disjoint from $U_{2 i}$ or is a singleton. Thus $\left|((V \times\{-1\}) \times\{2 i\}) \cap \psi\left(U_{2 i}\right)\right| \leqq 1$ and it follows from the statements in the proof of Lemma 10 that $(V \times\{-1\}) \times\{2 i\}$ is disjoint from any $\psi\left(U_{2 j}\right)$ (except if $\psi\left(U_{2 j}\right)$ is a singleton). Consequently, $(V \times\{-1\}) \times\{2 i\}$ would have to be covered by those $\psi\left(U_{2 j}\right)$ which are singletons. This is impossible however since there are only finite or countably infinite such $\psi\left(U_{2 j}\right)$ and $(V \times\{-1\})$ $\times\{2 i\}$ is uncountable.

A similar proof shows that $L_{1}$ is hopfian. This completes the proof of Theorem 2.

It can be observed that if $\left\langle X_{1} ; R_{1}\right\rangle$ and $\left\langle X_{2} ; R_{2}\right\rangle$ are hopfian graphs, then a free product of $M\left(X_{1}, R_{1}\right)$ and $M\left(X_{2}, R_{2}\right)$ is always hopfian. Therefore, there are $2^{\mathfrak{m}}$ pairs of pairwise nonisomorphic lattices of cardinality $m$ such that in any pair the lattices are hopfian and also their free product is hopfian. Also, for $m>\boldsymbol{N}_{0}$ we also have $2^{\mathrm{m}}$ pairs such that the lattices are hopfian but their free product is not. Both results are best possible. It would be interesting to prove the last statement also for $m=\aleph_{0}$.

\section{References}

[1] C. C. Chen and G. Grätzer, 'On the construction of complemented lattices', J. Algebra 11 (1969), 56-63.

[2] I. M. S. Dey and Hanna Neumann, 'The Hopf property of free products', Math. Z. 117 (1970), 325-339. 
[3] T. Evans, 'Finitely presented loops, lattices, etc. are hopfian', J. London. Math. Soc. 44 (1969), 551-552.

[4] G. Grätzer, Lattice Theory: First Concepts and Distributive Lattices (W. H. Freeman and Co., San Francisco, 1971).

[5] G. Grätzer, 'A reduced free product of lattices', Fund. Math. 73 (1971), 21-27.

[6] G. Grätzer, H. Lakser and C. R. Platt, 'Free products of lattices', Fund Math. 69 (1970), 233-240.

[7] G. Grätzer and J. Sichler, 'On the endomprophism semigroup (and category) of bounded lattices', Pacific J. Math. 35 (1970), 639-647.

[8] Z. Hedrlin and J. Sichler, 'Any boundable binding category contains a proper class of mutually disjoint copies of itself', Algebra Universalis 1 (1971), 97-103.

[9] P. Hell, 'Full embeddings into some categories of graphs', Algebra Universalis 2 (1972), 125-137.

[10] B. Jonsson, 'Sublattices of a free lattice', Canad. J. Math. 13 (1961), 256-264.

[11] H. Lakser, 'Simple sublattices of free products of lattices', manuscript.

[12] J. Sichler, 'Nonconstant endomorphisms of lattices', Proc. Amer. Math. Soc. 34 (1972), 67-70.

[13] J. Sichler, 'An example of a pair of hopfian groups whose free product is not hopfian', manuscript.

[14] P. M. Whitman, 'Free lattices I and II', Ann. Math. 42 (1941), 325-330; 43 (1942), 104-115.

Department of Mathematics

University of Manitoba

Winnipeg, Manitoba R3T 2N2

Canada 